\title{
Strategien zur Vermeidung von Burnout bei Ärzten
}

West CP. Dyrbye LN. Erwin PJ. et al. Interventions to prevent and reduce physian burn out: A systematic review and metaanalysis. Lancet 2016; 388: $2273-2281$

Burnout wird von der Psychologin C. Marflach definiert als Zustand, in dem Ärzte eine Erosion an Werten, Würde, Geist und Motivation in ihrer Arbeit erleben. Die dabei auftretende sogenannte Depersonalisation meint eine zynische Grundhaltung und fehlende innere Verbindung zum Inhalt der Medizin, dem Wohl der Patienten.

In diesem systematischen Review und dieser Metaanalyse wurden Interventionen zur Prävention und Reduktion des ärztlichen Burnouts ausgewertet. Insgesamt wurden 15 randomisierte Studien mit 716 Ärzten und 37 Kohortenstudien mit 20914 Ärzten ausgewertet. Der Burnout konnte präventiv von 54 auf $44 \%$ reduziert werden, der Erschöpfungsscore von 23,8 auf $21,2 \%$ und der Depersonalisationsscore von 9,1 auf 8,4. Eine schwergradige emotionale Erschöpfung konnte von 38 auf $24 \%$ reduziert werden und eine besonders schwergradige Depersonalisation von 38 auf $34 \%$.

Insgesamt zeigten sich sowohl individuelle Ansätze als auch strukturelle bzw. organisatorische Strategien zur Prävention des Burnouts erfolgreich.

In der Diskussion der Ergebnisse weisen die Autoren darauf hin, dass die Datenlage schmal ist. Weder ist geklärt, welche Strategien zu bevorzugen sind, noch wie diese aufeinander bezogen werden können, um ein besseres Ergebnis zu erreichen.

\section{Kommentar}

Was ist Burnout? Handelt es sich um ein individuelles Dispositionsrisiko, d.h. um eine Krankheitsneigung oder um Konsequenz einer systematischen Überforderung, die strukturell vorgegeben und für das Individuum nicht beherrschbar ist? Tatsache ist, dass das Burnout unter Ärzten ein zunehmendes Problem darstellt. „Physician burnout has reached epidemic levels": Im begleitenden Editorial von R. M. Eppstein und M. R. Trivitera wird festgestellt, dass der Burnout potenziell heute mehr als die Hälfte der praktizierenden Ärzte in den USA betrifft und in seinem Ausmaß die Funktionalität des medizinischen Systems in Frage zu stellen beginnt.

Diese Analyse zeigt, dass es eine Reihe von begrenzt erfolgreichen Strategien gibt, dem Burnout präventiv zu begegnen, dazu gehören Selbstwahrnehmung bzw. Selbstreflektion, Resilienz, Gespräche über den eigenen Standort und die Perspektiven. Die Betrachtung des Burnouts nur als individuelles Problem wahrzunehmen, wird jedoch nicht ausreichend sein.

Der Burnout kann gesehen werden als ein Symptom der zunehmenden Abschaffung des Ärztlichen im Rahmen eines rein wirtschaftlich aufgestellten Medizinbetriebs, einer Abschaffung freilich, an der die Ärzte selbst keinesfalls unschuldig sind. Ohne eine umfassende Reflexion der Grundlagen und Ziele der Medizin und der Rolle der Ärzte sowie der Patienten wird es keine Aussicht geben, dem Problem des Burnouts Herr zu werden. Niemand aber kann die Not wenden, wenn dies nicht die Ärzte bzw. ihre Interessensgruppen selbst in die Hand nehmen und in die Öffentlichkeit tragen.

Prof. Santiago Ewig, Bochum 